\title{
Serious Adverse Events Reporting and Follow-Up Requirements in the European and Developing Countries Clinical Trials Partnership-Funded Clinical Trials: Current Practice
}

\author{
Jean Marie Vianney Habarugira ${ }^{1 *}$, Antonia Agustí ${ }^{2}$ and Michael Makanga1 \\ ${ }^{1}$ European and Developing Countries Clinical Trials Partnership (EDCTP), The Hague, The Netherlands \\ ${ }^{2}$ Autonomous University of Barcelona, Barcelona, Spain
}

\begin{abstract}
Background: This study aimed to assess the current pharmacovigilance practice and compliance with the International Committee for Harmonization Good Clinical Practice (ICH-GCP) requirements within a range of clinical trials funded by the European and Developing Countries Clinical Trials Partnership (EDCTP).

Methods: A combination of a retrospective desk review of projects documentation as of June 2014 and a prospective survey among EDCTP-funded clinical trials investigators were used.

Results: The overall survey response rate was $64.3 \%$. Among the 54 trial investigators who responded to the survey, $64 \%$ are sponsored by academic institutions, $25 \%$ by public research institutions, and $6 \%$ by Product Development Partnerships (PDPs). $77 \%$ of the Sponsors are based in Europe, $17 \%$ in Africa and $6 \%$ are globalbased institutions. $75 \%$ of the respondents confirmed occurrence and reporting of Serious Adverse Events (SAEs) in their trials. The primary reference document for SAEs reporting and follow up reported as \% of clinical trials are clinical trial protocols $(81.5 \%)$, SOPs for handling SAEs $(50 \%)$ and investigator brochure $(11 \%)$. The average SAE reporting time by $81 \%$ of respondents within 24 hours, $11 \%$ within 48 hours, $4 \%$ within 7 days and $2 \%$ between 7 and 15 days. Majority (79.6\%) of investigators report SAEs directly to the their trial Sponsors, $62.3 \%$ to National Ethics Committees (NECs) and Institutional Review Boards (IRBs), 33.3\% to the national regulatory authorities (NRAs), $22.2 \%$ to the safety monitors, and $3.7 \%$ to the Contract Research Organisations (CROs). Combinations of these recipients were reported by several respondents. Among the 41 respondents who reported SAEs occurrence in their trial, only 22 confirmed that they are required to report to their NECs and IRBs. $85 \%$ of respondents send SAEs reports by e-mail, $27.8 \%$ by Fax mail, $11.1 \%$ by telephone and $9.3 \%$ as printed hard copies.

Conclusion: A majority of respondents $(75 \%)$ confirmed that SAEs have occurred in their trials; in this group, $45 \%$ of respondents did not confirm the requirement of reporting SAEs to local oversight bodies. It is important that both NECs and NRAs in all countries where clinical trials are conducted clearly make available their reporting requirements to the investigators to ensure adequate compliance with local reporting requirements. Further studies are necessary to better understand the magnitude of this problem and to strengthen capacity of local trial oversight by NECs and NRAs.
\end{abstract}

Keywords: Pharmacovigilance; Drug safety; Serious adverse events; Poverty-related diseases; Sub-Saharan Africa; EDCTP; Eu2P

Abbreviations: CIOMS: Council for International Organizations of Medical Sciences; CRO: Contract Research Organization; DSMB: Data and Safety Monitoring Board; EDCTP: European and Developing Countries Clinical Trials Partnership; Eu2P: European programme for Pharmacovigilance and Pharmacoepidemiology; GCP: Good Clinical Practice; HIV/AIDS: Human immunodeficiency virus and acquired immunodeficiency syndrome; IB: Investigator Brochure; ICH: Institutional Committee for Harmonization; IRB: Institutional Review Board; NEC: National Ethics Committee; NRA: National Regulatory Authorities; PDP: Product Development Partnership; SAE: Serious Adverse Event; SOP: Standard Operating Procedures; SSA: SubSaharan Africa

\section{Introduction}

As part of the broader regulatory spectrum, Pharmacovigilance is a complex process with phases that include data collection, data management, signal detection, safety issues assessment, decision making, communication and action [1]. In this process, the role of pharmaceutical companies as Marketing Authorization Holders (MAH) is well defined. Therefore, in industry-sponsored trials, MAH have Pharmacovigilance units with the knowledge and resources to efficiently deal with the process from data collection to taking action.
However, in trials sponsored by academic institutions or PDPs the requirements to Sponsor are defined, but the capacity to deal with pharmacovigilance tasks is not well documented.

There have been a growing number of partnerships or alliances between academia, pharmaceutical industry and other stakeholders such as funding agencies [2]. The growing alliance between academia and the pharmaceutical industry has given rise to serious concerns over issues that include poor reporting and management of adverse events [3]. These concerns are observed in developing countries where a substantial increase in the number of clinical trials has taken place in recent years.

*Corresponding author: Jean Marie Vianney Habarugira, European and Developing Countries Clinical Trials Partnership. Anna van Saksenlaan 51, $2593 \mathrm{HW}$, The Hague, The Netherlands, Tel: +31 (0)70 34408 91; E-mail: habarugira@edctp.org

Received October 10, 2014; Accepted November 20, 2014; Published November 27, 2014

Citation: Habarugira JMV, Agustí A, Makanga M (2014) Serious Adverse Events Reporting and Follow-Up Requirements in the European and Developing Countries Clinical Trials Partnership-Funded Clinical Trials: Current Practice. J Pharmacovigil 2: 148. doi:10.4172/2329-6887.1000148

Copyright: ( 2014 Habarugira JMV, et al. This is an open-access article distributed under the terms of the Creative Commons Attribution License, which permits unrestricted use, distribution, and reproduction in any medium, provided the original author and source are credited. 
As opposed to industry sponsored trials, academic or PDPsponsored trials rely on grants money to ensure PV activities are appropriately carried out. Funding agencies such as EDCTP can play an important role in ensuring academic institutions with limited funding, are encouraged to generate and share safety data. In 2007, Pirmohamed et al. [4] argued that the lack of local expertise in pharmacovigilance could be tackled through developing exchange programmes with major drug regulatory agencies and sharing best practices. In these academia-sponsored trials, European institutions have limited periods of involvement depending on the amount of available funding. The resource-limited settings where the trials are conducted are weak in terms of pharmacovigilance systems. According to Bakare et al. [5] for instance in the area of HIV, previous efforts to address the need for better drug safety monitoring systems for ARVs in resource-limited settings have had varying success, and this emphasizes again the need to mobilize resources and knowledge in the area of pharmacovigilance. Stergachis et al. [6] conducted a situational analysis of pharmacovigilance plans in the global funds and US president's malaria initiative proposals concluded that there were few requests for funding for pharmacovigilance activities which demonstrated a lack of emphasis on pharmacovigilance systems in grants recipient countries.

Several initiatives are being implemented to improve Pharmacovigilance systems, and health research funding agencies such EDCTP and like-minded organizations will have to engage in a close collaboration with these existing structures to work towards the improvement of pharmacovigilance systems.

One of these initiatives working on the improvement of pharmacovigilance systems in sub-Saharan Africa is the University of Ghana Medical School [7] which has been designated in 2009 as a WHO Collaborating Centre for Advocacy and Training in Pharmacovigilance. Since its implementation, the centre has been active in sub-Saharan Africa advocating and training personnel in Pharmacovigilance and providing technical support to assist countries and institutions in improving their pharmacovigilance systems.

In 2003, the European Parliament and Council co-decided on the European Commission supporting a long-term partnership between Europe and developing countries for the development of new medicines and vaccines against the Human Immunodeficiency Virus /Acquired Immunodeficiency Syndrome (HIV/AIDS), malaria and tuberculosis [8]. In this context, EDCTP launched its first set of calls for proposals in 2004, resulting in 65 calls over a 10 years period. From these calls, 100 clinical trials and a significant number of capacity building activities have been funded with the main objective of accelerating the development of new or improved drugs, vaccines, microbicides and diagnostics against HIV/AIDS, tuberculosis and malaria. EDCTP funds non-commercial clinical trials and the Sponsors for these trials are a combination of academic and non-academic product development partners including private pharmaceutical companies, with a broad diversity of experience and competence in this area.

All these clinical trials must obtain ethical and regulatory approval from all countries where they are actually conducted.

According to the ICH-GCP guidelines, all SAEs must be reported immediately to the sponsor except for those SAEs specifically stipulated in the protocol or other documents (e.g., investigator's brochure) as not requiring immediate reporting. The investigator should also comply with the applicable regulatory requirements related to the reporting of unexpected SAEs to the NRAs and IRBs [9]. Moreover, assessment of safety is one of the endpoints the trial protocol should include in evaluation of investigational products prior to registration, and for registered products being optimized for use in special patient populations for purposes of label extension. Depending on the type of trial, the protocol has to provide definitions and categorization of SAEs. The protocol has to clearly indicate what, how, when and to whom SAEs are reported. If these elements are not clarified in the protocol, the Sponsor has to ensure the investigators are provided with other relevant reference documents. To ensure that what is stated in the clinical trials protocols is actually implemented; Standard Operating Procedures (SOPs) should be developed for specifically handling reporting of SAEs. Additionally, compliance to the trial protocol and adherence to SOPs should be monitored by both internal and external clinical monitors commissioned by the clinical trials Sponsors.

Pharmacovigilance plans rely on pre-marketing data to identify risk drivers and concerns requiring further monitoring in the postmarketing phase [10], however there is a lower SAEs reporting rate observed in post-marketing observational studies using passive pharmacovigilance monitoring compared to active data collection of AEs during pre-marketing clinical trials [11]. Hence, there is a need to evaluate how the funded trials are contributing to the active SAEs reporting in SSA countries. The trials involved different types of populations - adults, adolescents, children, infants, pregnant women. Based on the figures reported by completed trials and looking at the sample sizes stated in protocols of ongoing trials, we estimated the total number of participants in the 100 clinical trials at more than 93,000 individuals.

Collection of safety data is a continuous process and Pharmacovigilance in SSA has been progressively strengthened by EDCTP through the funding of trials and other related activities (e.g. ethics and regulatory training). Reporting of safety data contributes enormously to good Pharmacovigilance. With such a coverage of different population groups, with different disease, using a range of different medicinal products, the amount of safety data collected and reported by EDCTP-funded investigators is one of the most important deliverables and contributions to the available knowledge on safety of medicinal products used for the treatment and prevention of the three major poverty-related diseases (HIV/AIDS, tuberculosis and malaria). In a study published in 2011 by Kuemerle et al. [12] the authors report that regarding safety of arteminisin-based combination therapy (ACT), adverse drug reactions data came predominantly from non-endemic countries.

In 2010, upon request by the Global Fund, a consultative process was initiated by the WHO and other agencies, with the aim of producing a pharmacovigilance strategy for use by countries that were seeking to advance their Pharmacovigilance systems with support from the Global Fund or similar agencies. One of the achievements of this consultative process was the agreement on a set of minimum requirements [13] for a functional national pharmacovigilance system. These requirements included a clear communication strategy for routine and crisis communication.

Many developing countries, including those in SSA, lack sufficient infrastructure to assess causality, evaluate the incidence and risk factors of adverse events, make meaningful comparisons with prior experience or calculate the risks attributable to adverse events [14].

According to an analysis conducted in 2012 looking at the spontaneous reports sent to VigiBase, on average high-income countries generate 130 ADR reports per million inhabitants per year, lower middle-income countries produce 12 annual reports per million 
Citation: Habarugira JMV, Agustí A, Makanga M (2014) Serious Adverse Events Reporting and Follow-Up Requirements in the European and Developing Countries Clinical Trials Partnership-Funded Clinical Trials: Current Practice. J Pharmacovigil 2: 148. doi:10.4172/2329-6887.1000148

Page 3 of 8

inhabitants and low-income countries produce 3 annual reports per million inhabitants [15].

EDCTP-funded trials are carried out in settings where pharmacovigilance systems are poor and funding for pharmacovigilance related activities is limited. There is therefore a need for EDCTP and similar organizations to evaluate the quantity and quality of safety data coming from the areas in which their-funded trials are carried out.

\section{Objectives}

The overall aim of this study was to assess the current practice and compliance with ICH-GCP requirements in the detection, collection, assessment and reporting of SAEs in EDCTP-funded clinical trials.

The specific objectives of this study are as follows:

- To review and analyse documents used by EDCTP trials investigators for SAEs reporting and follow up;

- To perform a cross category gap analysis of the compliance with the ICH-GCP requirements on safety reporting.

\section{Methods}

\section{Desk review and survey}

This study was implemented through two step-wise and complementary approaches to address the key questions in order to achieve the study objectives, while being cognisant of the limited study duration.

First, a retrospective desk review was done mainly focusing of the study protocols. This aimed at establishing what was planned to be done in the handling of SAE.

Second, a prospective survey was designed and implemented targeting EDCTP-funded investigators. This approach aimed at getting more accurate and complete information, on what is actually practised. The survey questionnaire targeted clinical investigators or other key staff in the trials. The questions in the survey enabled a collection of accurate data on how the EDCTP- funded clinical investigators are dealing with the challenges of SAEs reporting and their follow up.

The desk review focused on 100 clinical trials funded by EDCTP in the period between 2004 and 2013. The first step of the desk review was to ensure the documentation available at the EDCTP office is sufficient to enable collection of the data needed to answer the questions raised in this study. The desk review was also used to establish the characteristics of the EDCTP-funded trials in terms of disease categories, studied interventions and trials phase. It was also used to establish the profile of the institutions involved as Sponsors of EDCTP-funded trials.

The survey targeted investigators and other key staff of the 84 trials selected for the study. Survey questionnaires (Table 1) were sent to the potential respondents and follow-up was done in the period of March and April 2014.

\section{Data reconciliation and analysis}

Survey responses were reconciled with data from the desk review and each of the questions here below was analyzed in the context of the disease area, the studied intervention and the type of Sponsor.

The desk review enabled us to categorize the trials based in following characteristics:

- Disease area: HIV/AIDS, tuberculosis and malaria;

- Studied intervention: drugs, vaccines, microbicides and diagnostics;

- Clinical trial phase: I, II, III and IV;

- Trial Sponsor type: academic, industry, public research institution, product research development ;

- Trial Sponsor location: Africa, Europe, Global beyond Africa and Europe.

The 54 returned questionnaires were checked for completeness and a check for accuracy of unique information (i.e. trial title or grant code etc.) was performed. Accuracy of the received response against guidelines of each Sponsor was not checked, therefore, the analysis focused only on the response provided by the investigators. Descriptive formulas from Excel were used to the determine the level to which SAEs occurred and were reported, the types of documents which are referred to during SAEs reporting, causality assessment and follow up, the timeframe in which initial reports had to be submitted, the types of recipients to which reporting was required, the mode used to transmit the reports and the level to which the role of safety monitors was understood.

\section{Results}

Among the 100 clinical trials funded by EDCTP are 30 trials on HIV/AIDS, 34 on malaria, 27 on tuberculosis and 9 trials focusing on HIV/AIDS with tuberculosis co-infection.

Because this study focused on SAEs handling in trials which evaluated medicinal products, 84 of the 100 clinical trials were included in this study. As shown in Figure 1, 16 trials were excluded from the study for the following reasons:

- Eleven trials were developing diagnostics tools;

- In three trials there was no medical product involved (e.g. use of medical technologies to assess treatment adherence);

- For one trial, the protocol was still being developed at the time of the desk review;

- One trial had been prematurely terminated.

1. What was/is the primary source of guidance on SAEs reporting and follow up?

2. Which document (s) did/do you refer to for SAEs reporting timelines?

3. Which document(s) did/do you refer to for the SAEs causality assessment?

4. Were there SAEs occurrences during the conduct of your trial?

5. To whom were/are you required to send initial and follow up SAEs reports?

6. What was/is the mode used to send SAEs reports?

7. Within what timeframe did/do you have to send initial SAE reports?

8. Within what timeframe was/is the Sponsor required for respond to initial SAEs reports?

9. What was/is the role of trial monitors in the SAEs reporting and follow-up?

10. Prior to protocol approval, did/do you have to provide (to ethics and regulatory bodies) details on how SAEs will be reported and followed up? 


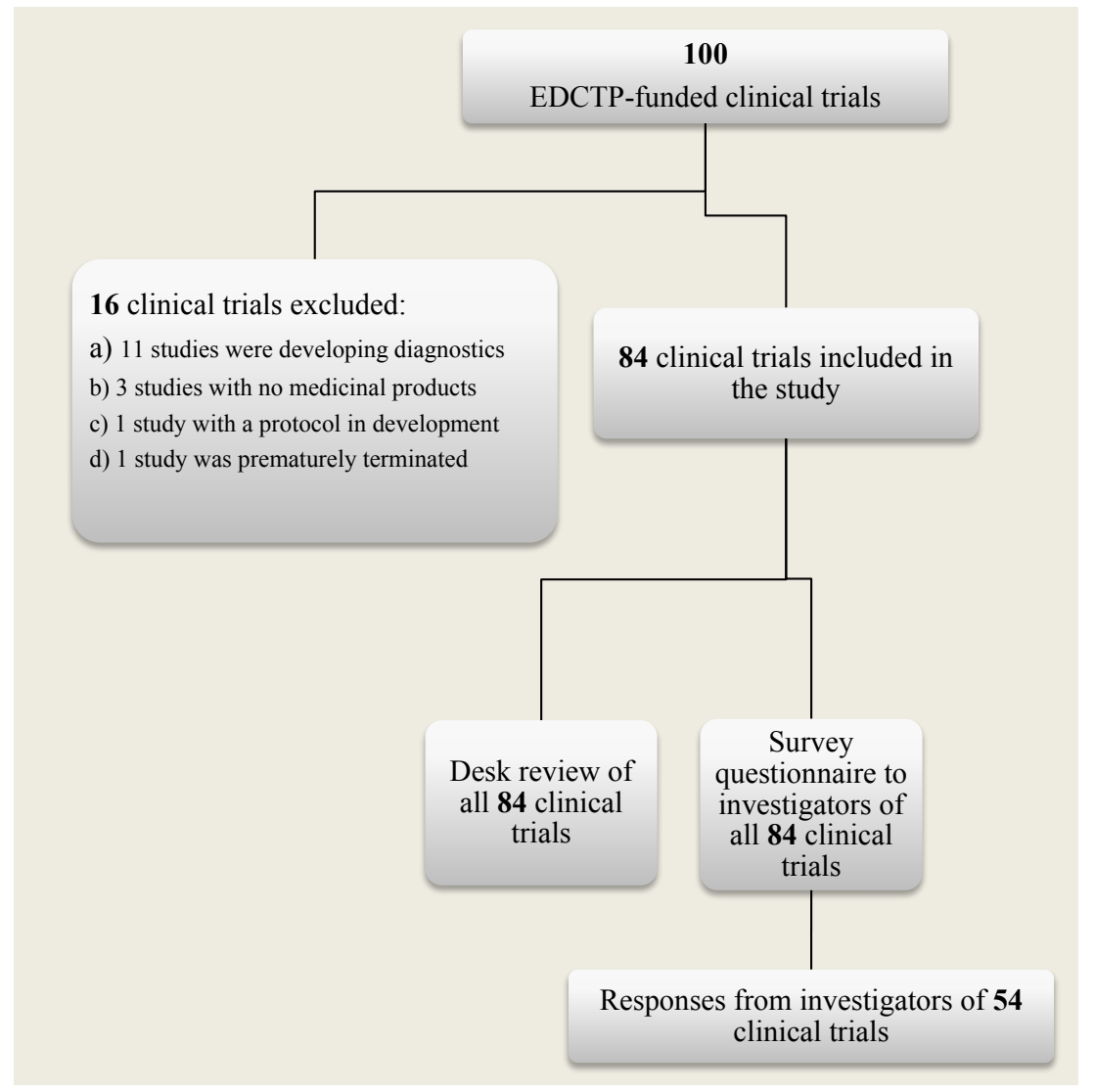

Figure 1: Selection of trials included in the desk review and survey.

\begin{tabular}{|c|c|c|c|c|}
\hline \multirow{2}{*}{ Disease area } & \multicolumn{3}{|c|}{ Studied Interventions } & \multirow{2}{*}{ Total } \\
\cline { 2 - 5 } & Drugs & Microbicides & Vaccines & \\
\hline HIV & 17 & 2 & 8 & $\mathbf{2 7}$ \\
\hline Malaria & 22 & 0 & 11 & $\mathbf{3 3}$ \\
\hline TB & 10 & 0 & 6 & $\mathbf{1 6}$ \\
\hline TB/HIV & 8 & 0 & 0 & $\mathbf{8}$ \\
\hline Total & $\mathbf{5 7}$ & $\mathbf{2}$ & $\mathbf{2 5}$ & $\mathbf{8 4}$ \\
\hline
\end{tabular}

Table 2: Disease area and intervention categories in the 84 trials.

\section{Profile of the clinical trials included in the study}

Among the 84 trials selected for the study, 57 trials are developing drugs, and 25 trials are developing vaccines, while 2 are focusing on HIV microbicides. In terms of disease categories, the 84 trials are divided into 33 malaria trials, 27 HIV/AIDS trials, 16 tuberculosis trials and 8 trials on HIV/AIDS with tuberculosis co-infection (Table 2).

EDCTP's main focus is to fund phase II and III trials, but a significant number of trials in phase I and IV have been supported as well. Most of the funded drug trials are in phase II (19 trials), phase III (18 trials) or phase IV (17 trials). The majority of the vaccine trials are in phase I (12 trials) and II (9 trials). It is worth noting that majority of the phase IV studies are testing optimisation of registered products for purpose of label extension in special patient groups such as pregnant mothers, children and infants, and patients with co-infections.

\section{Trials Sponsorship}

EDCTP-funded trials are carried-out in partnership between
European and African research institutions. Capacity development is an important component of these partnerships and this involves also the strengthening of the pharmacovigilance systems in countries where the studies are conducted. These trials are funded through grants to non-for profit institutions, (e.g. Universities or PDPs), based in Africa or in Europe. The performance of these Sponsors is difficult to quantitatively assess but in this study, through the desk review we tried to characterize these Sponsor institutions in terms of their location (e.g. Europe, Africa) and type (e.g. academic, pharmaceutical industry).

Based on the results from the desk review, we noted that although all EDCTP-funded trials are conducted in SSA Africa, a large majority (77\%) of these trials are sponsored by Europe-based institutions. As shown in Figure 2, about only $17 \%$ and $6 \%$ are sponsored by Africabased and global-based institutions, respectively.

It is important to note that in categorizing the trials Sponsors we distinguished Universities from other public research institutions with funding for specific research areas. As shown in Figure 3, $64 \%$ of EDCTP-funded trials with medicinal products are sponsored by Academic institutions, while $25 \%$ are sponsored by public research institutions and $6 \%$ by PDPs. One trial was sponsored by the World Health Organization (WHO), one other trial by a private entity and two trials were carried out without formalized sponsorship status with the hosting institutions. The survey questionnaire was sent to the investigators or key staff of the 84 selected clinical trials. In total, we received completed questionnaires from 54 of the 84 clinical trials, corresponding to a $64.3 \%$ response rate. Because the final analysis 


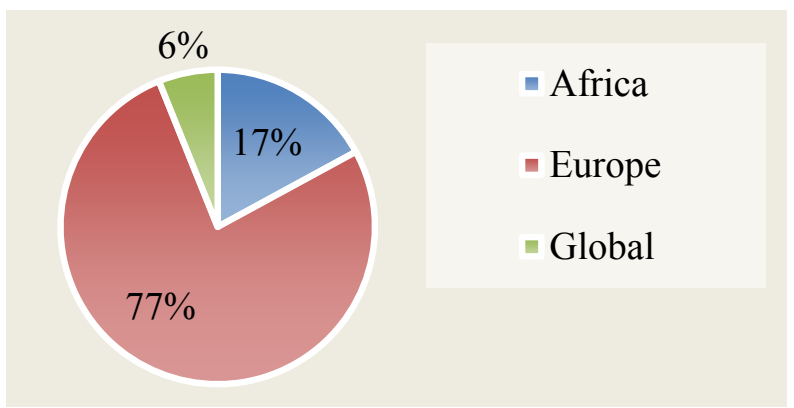

Figure 2: Location of trial Sponsors of all 84 trials.

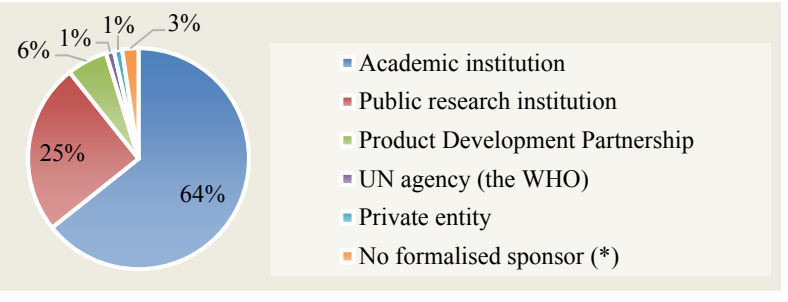

$\left.{ }^{*}\right)$ There was no evidence of a formalized Sponsor for two trials Figure 3: Types of Sponsors in the 84 trials.

\begin{tabular}{|c|c|c|c|c|}
\hline \multirow{2}{*}{ Type of Sponsor } & \multicolumn{3}{|c|}{ Occurrence of SAEs } & \multirow{2}{*}{ Total } \\
\cline { 2 - 4 } & Cannot remember & No & Yes & \\
\hline Academic & & 11 & 23 & 34 \\
\hline PDP & & & 2 & 2 \\
\hline Public research institution & & & 15 & 15 \\
\hline UN agency & 1 & & & 1 \\
\hline No formalized Sponsor & & 1 & 1 & 2 \\
\hline Total & $\mathbf{1}$ & $\mathbf{1 2}$ & $\mathbf{4 1}$ & $\mathbf{5 4}$ \\
\hline
\end{tabular}

Table 3: Occurrence of SAEs per type of Sponsor.

focused on the survey responses, we analysed the profile of the trials whose investigators responded to the survey, to ensure all categories of disease area, studied interventions and Sponsor type were represented.

The disease-intervention profile shows that among the 54 received responses, 19 were from HIV/AIDS trials investigators, 22 from malaria investigators, 10 from tuberculosis and 3 from TB/HIV co-infections researchers. 23 responses came from drug trials investigators, 21 from vaccines trials investigators and one from microbicide trial. Among the trials whose investigators responded to the survey, a majority $(80.8 \%)$ were sponsored by Europe-based institutions, while only $13.5 \%$ and $5.8 \%$ are sponsored by African and global-based institutions, respectively.

A large number (65.4\%) of the investigators who responded to the survey are sponsored by academic institutions, about $28.8 \%$ of the trials are sponsored by public research institutions. 3.8\% of the respondents are sponsored by PDPs while 1 trial (1.9\%) was sponsored by the WHO.

\section{SAEs occurrence and reporting}

Through the survey an attempt was made to estimate the magnitude of SAEs occurrence in EDCTP-funded clinical trials. The survey responses confirmed that SAEs occurred and were reported in $41(75 \%)$ of the 54 clinical trials whose investigators responded to this question. Albeit linking SAEs with causality was beyond the scope of this study, the occurrence and reporting of SAEs at such level underpins the importance of these clinical trials towards generation of pharmacovigilance data on evaluated medicinal products in pre- or post-marketing stages. One of the respondents did not recall if SAEs occurred in the trial (Table 3 ).

\section{Reference documents for SAEs reporting and follow up}

The above described results show that SAEs occurred in $75 \%$ of the EDCTP-funded trials whose investigators responded to the survey. Another question of the survey sought to know the primary reference document used by the investigators for SAEs reporting and follow up. As shown in Table 4, among the 54 survey respondents, 43 (81.5\%) mentioned the trial protocol. However, an important number of respondents (50\%) included trial SOPs on SAEs reporting among their primary source of guidance. The Investigator Brochure was mentioned by $11.1 \%$ of the respondents. Other respondents mentioned study manuals (7.4\%), ICH guidelines (3.7\%), National guidelines $(3.7 \%)$, CIOMS guidelines (1.9\%), trial safety plan (1.9\%) and severity grading scales (1.9\%).

When the question was specified to understand what the reference for causality assessment was, the clinical trial protocol was still mentioned by a majority of the respondents but on a lower percentage in comparison to the number of those who considered the trial protocol to be the primary source of guidance for SAEs reporting and follow-up. About $61.1 \%$ of the respondents mentioned the clinical trial protocol while $33.3 \%$ confirmed that they use clinical trial SOPs as reference for causality assessment.

To this same question, the Investigator Brochure was mentioned by $27.8 \%$ of the respondents. As shown in Figure 4, it is important to note that beside the above mentioned main sources of reference for causality assessment, smaller percentages of the respondents mentioned other references such as the Sponsor's or WHO's grading scales (5.6\%), Summary of Product Characteristics or SmPC (5.6\%), relevant publications (3.7\%), medical textbooks (3.7\%), communications with drug manufacturer, SAEs databases and Case Report Forms.

\section{Timeframe for initial SAE reports submission}

On the question of timeframes to submit initial SAEs reports, a vast majority ( $81 \%)$ of the survey respondents confirmed that SAEs have to be reported within 24 hours of the investigator becoming aware of the event, $11 \%$ have to report SAEs with 48 hours, while a smaller group of investigators have to report within 7 days (4\%) or in an interval of 7 to 15 days (2\%) (Figure 5).

\section{Recipients of SAEs reports}

As shown in Table $5,79.6 \%$ of the 54 respondents indicated that

\begin{tabular}{|c|c|c|}
\hline Mentioned reference & Number of responses & \% (Total N=54) \\
\hline Trial protocol & 44 & $81.5 \%$ \\
\hline Trial SOPs & 27 & $50.0 \%$ \\
\hline Investigator's Brochure & 6 & $11.1 \%$ \\
\hline Study Manual & 4 & $7.4 \%$ \\
\hline ICH guidelines & 2 & $3.7 \%$ \\
\hline National guidelines & 2 & $3.7 \%$ \\
\hline CIOMS guidelines & 1 & $1.9 \%$ \\
\hline Trial safety plan & 1 & $1.9 \%$ \\
\hline Severity grading scale & 1 & $1.9 \%$ \\
\hline
\end{tabular}

Table 4: Primary source of guidance on SAEs reporting and follow up. 


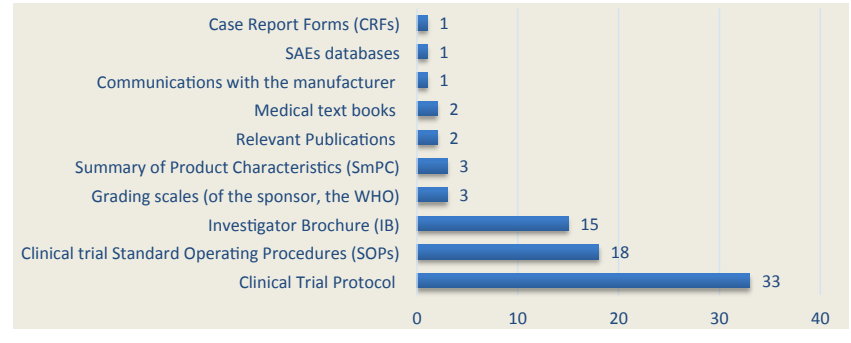

Figure 4: References for causality assessment.

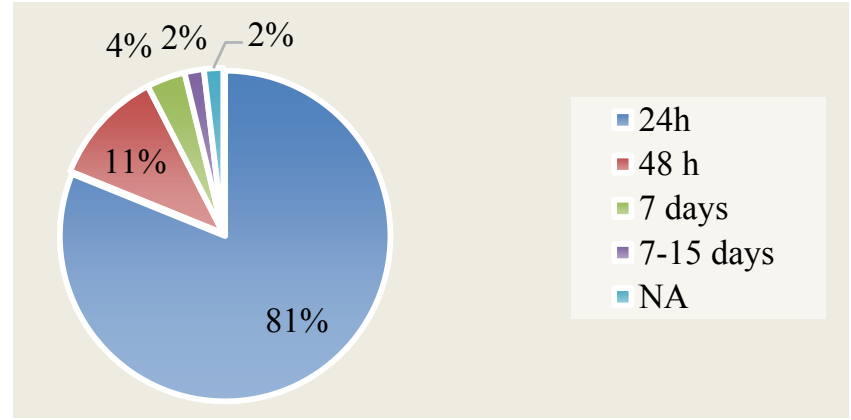

Figure 5: Timeframe for sending initial SAE report.

\begin{tabular}{|c|c|c|}
\hline To whom are SAEs reports sent & $\begin{array}{c}\text { Number of time } \\
\text { mentioned }\end{array}$ & \% (total $\mathbf{n = 5 4 )}$ \\
\hline Trial Sponsor & 43 & $79.6 \%$ \\
\hline DSMB & 31 & $57.4 \%$ \\
\hline NEC or IRB & 33 & $62.3 \%$ \\
\hline Regulatory Authority & 18 & $33.3 \%$ \\
\hline Safety Monitor & 12 & $22.2 \%$ \\
\hline Contract Research Organization & 2 & $3.7 \%$ \\
\hline
\end{tabular}

Table 5: Required recipients of SAEs reports.

they are required to send initial and follow up SAEs reports to the Trial Sponsor. There are chains of communication that are specific to each trial but, since not all investigators are required to send SAEs reports to their Trial Sponsor, it is important to know who they are required to send the reports to.

In their response, $57.4 \%$ of the 54 respondents mentioned the Data and Safety Monitoring Board (DSMB) which certainly regularly communicates with the Sponsor. $62.3 \%$ confirmed that they are required to send the reports to their NECs or IRBs, $33.3 \%$ mentioned the regulatory authorities, $22.2 \%$ mentioned the safety monitors, and a smaller number (3.7\%) of respondents mentioned the CRO.

Local reporting is crucial for these trials which are sponsored mainly by external institutions. However, on the list of required recipients of SAEs reports, Ethic Committees or IRBs were mentioned by $62.3 \%$ of the respondents and 33.3\%\% included Regulatory Authorities. Among the 41 investigators who confirmed that SAEs occurred in their trial, not all but only $22(55 \%)$ confirmed that they are required to report the SAEs to their ECs or IRBs. Out of the 41 investigators who confirmed SAEs occurrence in their trials, the 22 investigators who confirmed the requirement of reporting to NECs and IRBs were linked to Sponsors categorised as follows:
- 5 (out of 5) Africa-based Sponsors

- 16 (out 33) Europe-based Sponsors

- 1 (out 2) global-based Sponsor

- 15 (out of 23) academic institutions

- 1 (out of 2) PDP

- 8 (out of 15) public research institutions

Among the 41 investigators who confirmed SAEs occurrence in their trials, all 5 respondents linked to Africa-based Sponsors confirmed that they are required to report to their NECs or IRBs. However, only 16 of the 33 investigators linked to Europe-based Sponsors confirmed this requirement. Only 15 of the 23 investigators sponsored by academic institutions confirmed this requirement to report SAEs to local oversight bodies. According to the ICH-GCP guidelines (9), "the Sponsor may transfer some or all of the Sponsor's trial-related duties and functions to a CRO", and "any trial-related duty and function that is transferred to/and assumed by a CRO should be specified in writing". In relation to this, it is important to note the small but important percentage (3.7\%) of respondents who indicated that they are required to send initial and follow up SAEs reports to CRO.

\section{The mode used to send SAEs reports}

As shown in Figure 6, most of the surveyed investigators confirmed that they use e-mail for submission of SAEs reports. Among the 54 surveyed investigators, $46(85.2 \%)$ indicated that e-mail is used to submit SAEs reports, 15 (27.8\%) respondents mentioned fax, and 6 (11.1\%) respondents mentioned telephone, while hard copies as mode of SAEs reports submission was mentioned by $5(9.3 \%)$ respondents.

\section{The role of Safety Monitors}

According to the ICH-GCP guidelines (9), the monitors' responsibilities include "determining whether all AEs are appropriately reported within the time periods required by GCP, the protocol, the IRB and EC, the Sponsor and the applicable regulatory requirements".

In this study, through the survey we attempted to establish the roles of safety monitors in EDCTP-funded trials. The responses were very heterogeneous and qualitatively informative. This disclosed a capacity gap that needs to be bridged through training to ensure improved quality control of future clinical trials.

Examples of responses to the question: "What was/is the role of trial monitors in the SAEs reporting and follow-up?"

- Only "review during visits that SAEs were reported and completed as required".

- To "check that SAEs had been reported timeously, and had been dealt with appropriately (as per protocol and SOPs), check the clinical case reporting forms."

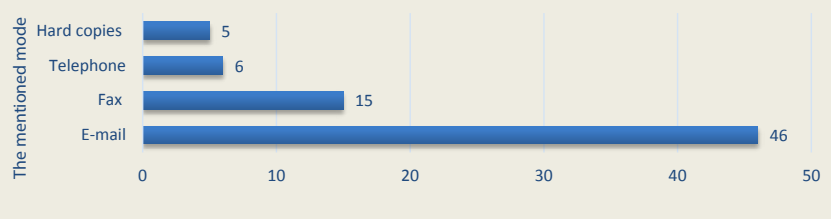

Figure 6: The mode used to send SAEs reports. 
- Monitors helped in ensuring timelines of reporting from the site, ensure final reports are sent, and reconcile the total number of SAEs"

- The trial monitors will review the patient files and ensure that all SAE's were reported to the regulatory bodies within the required timeframe and updated to a stable status.

- "The Local Safety Monitor provided real-time safety oversight. The Local Safety Monitor reviewed SAEs immediately after they occurred and followed the events until resolution. The Local Safety Monitor had the power to suspend enrolment into the study if deemed necessary following an SAE and immediately convene a meeting with the Sponsor and Principal Investigator. The Local Safety Monitor could convene a meeting with the Sponsor, PI or other parties involved at any time for further discussion as necessary."

\section{Discussion}

Overall the results of this study show that the current practice of active pharmacovigilance especially related to SAEs in EDCTP-funded clinical trials in greater than $70 \%$ of clinical trials complies with ICH requirements and has been enforced by the Sponsors. Some factors and the existing gaps related to limited systems and processes as well as human resources contributing in part to the non-compliance are revealed by this study.

The desk review revealed that most EDCTP-funded trials are sponsored by Europe-based academic or public research institutions. These institutions are very experienced in conducting academic research but have limited experience in conducting regulatory type of clinical trials. A small number of Africa-based universities and PDPs are involved as Sponsors of EDCTP-funded trials. Although pharmaceutical companies are involved in EDCTP-funded clinical trials, this analysis shows that no pharmaceutical partner is involved directly as a clinical trial Sponsor. The limited involvement of PDPs and non-involvement of pharmaceutical partners as clinical trial Sponsors may in part contribute to the lack of clearly defined SAEs handling procedures, where this gap exists.

The desk review results showed that most of the protocols contain a clear description on how to detect SAEs and collect relevant data, assess causality and report as appropriate. A large majority of the survey respondents confirmed that these trial protocols are indeed used as the primary reference for detection, assessment and reporting of SAEs occurring in EDCTP-funded trials. The survey also revealed other types of documents frequently used by the investigators for the handling of SAEs. For EDCTP and similar organizations funding clinical trials conducted in SSA, it is important to be informed on the reference documents used by investigators during the reporting and follow up of SAEs.

Moreover, the challenges related to SAEs reporting timelines, the compliance with ICH-GCP guidelines and the role of clinical monitors in ensuring quality control and assurance of compliance with the procedures and guidelines used in these clinical trials, require consideration. It is crucial that a critical mass of African clinical monitors with regulatory clinical trials expertise are trained and retained to work in Africa. Mentorship through hands on training and experience with pharmaceutical and product development partners is an approach that EDCTP in collaboration with WHO TDR is exploring [16]. Other pragmatic models of training such as reciprocal training of clinical monitors are also being explored $[17,18]$ by different research groups.

The study results show that among the 41 investigators who confirmed that SAEs occurred in their EDCTP-funded trial, only 22 confirmed that they are required to report the events to the local Ethics or Regulatory bodies. In this study, we did not look into specific requirements of each ethics committee and regulatory body, but it is very likely that institutional and/or national ethics and regulatory bodies require investigators to submit reports on SAEs occurring in the approved trials conducted in their territories.

A small percentage (3.7\%) of respondents indicated that they are required to send initial and follow up SAEs reports to CROs. EDCTP and similar organizations should be informed not only about the existence of such agreements but also about their content. Funders should be aware of the responsibilities of all involved parties including when tasks have been delegated or outsourced.

\section{Study Limitations}

Authors had limited access to documentation on trials that closed long before this study. Recall bias might have affected the study, especially in case of trials which were completed long before the conduct of our survey. The study was confined to clinical trials that received funding from EDCTP. Authors did not have direct feedback from ethics and regulatory bodies in countries where trials are conducted.

\section{Conclusion}

Clinical trial protocols and SOPs based on trial protocols are the most important reference used by EDCTP-funded investigators for reporting and follow-up of SAE. The SAEs reporting timelines requirements should be tightly monitored, and clarification in terms of SAEs reports recipients is needed to ensure local and international requirements are met.

A majority of respondents (75\%) confirmed that SAEs have occurred in their trials and in this group of respondents, $45 \%$ did not confirm the requirement of reporting SAEs to local oversight bodies. It is important that both NECs and NRAs in all countries where clinical trials are conducted clearly make available their reporting requirements to the investigators to ensure adequate compliance with local reporting requirements. These findings revealed a possible non-compliance with existing local reporting requirements or simply the absence of such requirements to report SAEs. This is an important gap in terms of requirements and compliance. Further studies are necessary to better understand the magnitude of this problem and to strengthen capacity of local trial oversight by NECs and NRAs

Despite their good academic research experience, the trial Sponsors (largely Europe-based academic institutions) have limited experience or resources to conduct regulatory type of trials. In SSA countries where EDCTP-funded trials are conducted, with limited safety monitoring capacity, there is a need to strengthen this capacity to ensure that both trial sponsors and investigators are adequately equipped with skills and experience to ensure compliance with all relevant international and national requirements.

\section{Acknowledgement}

We thank the European and Developing Countries Clinical Trials Partnership (EDCTP) for providing access to the data used in this study. We also thank the European Programme for Pharmacovigilance and Pharmacoepidemiology (Eu2P) for the academic supervision of the study. Special thanks go to all survey respondents for their time and valuable contribution. 
Citation: Habarugira JMV, Agustí A, Makanga M (2014) Serious Adverse Events Reporting and Follow-Up Requirements in the European and Developing Countries Clinical Trials Partnership-Funded Clinical Trials: Current Practice. J Pharmacovigil 2: 148. doi:10.4172/2329-6887.1000148

\section{References}

1. Fraunhofer Institute for Systems and Innovation Research (2006) Assessment of the European Community System of Pharmacovigilance: Final report. European Commission Enterprise and Industry Directorate-General, Unit F2, Pharmaceuticals.

2. Centre for International Drug Monitoring (2002) The Importance of Pharmacovigilance - Safety Monitoring of Medicinal Products. World Health Organization, Geneva.

3. Centre for International Drug Monitoring (2002) Chapter 4: Pharmacovigilance in Drug Regulation. The Importance of Pharmacovigilance - Safety Monitoring of Medicinal Products. World Health Organization, Geneva.

4. Pirmohamed M, Atuah K, Dodoo A, Winstanley $P$ (2007) Pharmacovigilance in developing countries. BMJ 335: 462-462.

5. Bakare N, Edwards I, Stergachis A, Pal S, Holmes C, Lindquist M, et al. (2011) Global Pharmacovigilance for Antiretroviral Drugs: Overcoming Contrasting Priorities. PLoS Med 8: e1001054.

6. Stergachis A, Bartlein RJ, Dodoo A, Nwokike J, Kachur SP (2010) A situationa analysis of pharmacovigilance plans in the Global Fund Malaria and U.S. President's Malaria Initiative proposals. Malar J 9: 148

7. Sulley A (2014) Brief History of Centre. WHO-CC for Advocacy \& Training in Pharmacovigilance.

8. European Commission - Research: the European Developing Countries Trials Programme initiative.

9. ICH Expert Working Group (1996) Guidelines for Good clinical practice E6 (R1). International Conference on Harmonisation of Technical Requirements for Registration of Pharmaceuticals for Human Use.
10. Mehta U, Allen E, Barnes K (2010) Establishing pharmacovigilance programs in resource-limited settings: the example of treating malaria. Expert Review of Clinical Pharmacology 3: 509-525.

11. Kabanywanyi AM, Mulure N, Migoha C, Malila A, Lengeler C, et al. (2010) Experience of safety monitoring in the context of a prospective observational study of artemether-lumefantrine in rural Tanzania: lessons learned for pharmacovigilance reporting. Malar J 9: 205

12. Kuemmerle A, Dodoo AN, Olsson S, Van Erps J, Burri C, et al. (2011) Assessment of global reporting of adverse drug reactions for anti-malarials, including artemisinin-based combination therapy, to the WHO Programme for International Drug Monitoring. Malar J 10: 57.

13. The Global Fund and the World Health Organisation (2010). Minimum requirements for a functional pharmacovigilance system.

14. WHO Global Advisory Committee on Vaccine Safety (GACVS), WHO secretariat (2009) Global safety of vaccines: strengthening systems for monitoring, management and the role of GACVS. Expert Rev Vaccines 8: 705-716.

15. Aagaard L, Strandell J, Melskens L, Petersen PS, Holme Hansen E (2012) Global Patterns of adverse drug reactions over a decade: Analyses of spontaneous reports to VigiBase. Drug Saf 35: 1171-1182.

16. EDCTP-TDR Fellowships - 2014

17. Chilengi R, Ogetii G, Lang T (2010) A Sensible Approach to Monitoring Trials: Finding effective solutions in-house. WebmedCentral Clinical Trials 1 : WMC00891.

18. Chantler T, Cheah PY, Miiro G, Hantrakum V, Nanvubya A, E et al. (2014) International health research monitoring: exploring a scientific and a cooperative approach using participatory action research. BMJ Open 4: e004104-e004104 